\title{
Poly(thiaheterohelicene): A Stiff Conjugated Helical Polymer Comprised of Fused Benzothiophene Rings
}

Synthesis of Heterocycles

Polymer-

Supported Synthesis

Key Words

thiaheterohelicene

intramolecular

ring-closing

stereoselectivity

helicity

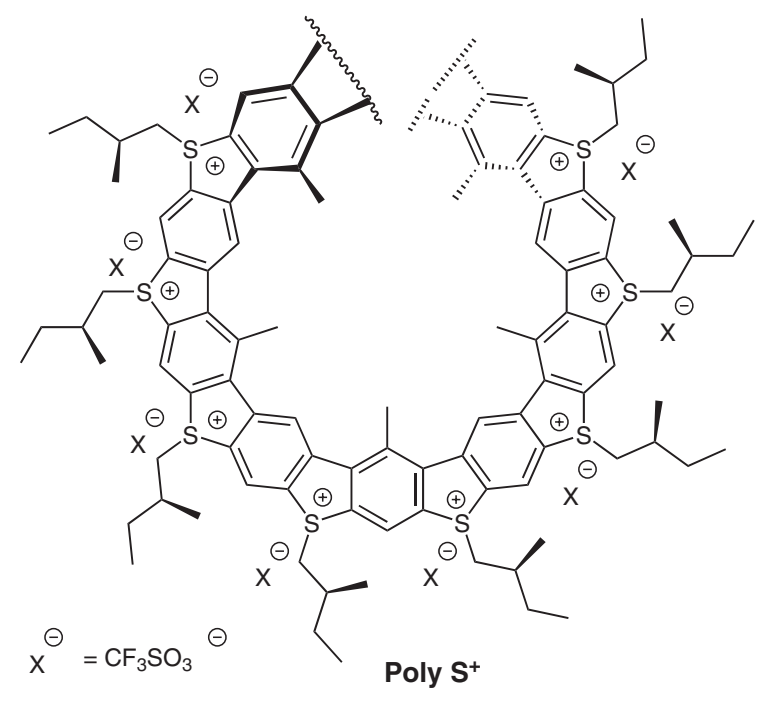

Significance: The first efficient asymmetric synthesis of helical heteroaromatic polymer comprised of fused benzothiophene rings in good chemical yield and stereoselectivity by intramolecular ring-closing condensation starting from inexpensive commercially available starting materials is reported. The synthetic materials form up to 12 helical pitches in the helical structure which is maintained over a wide range of temperatures in a variety of solvents. Chemo-physical properties of the new polymers are described.
Comment: Helical macromolecules are attracting attention as a result of their unusual physicochemical properties. Such compounds may find application in chiral separation and recognition in liquid crystals or may serve as chiral catalysts for asymmetric synthesis. Fused helical benzothiophene cores of this type may also be good candidates for organic conductive materials, organic molecular-based electromagnets, and nanometer-sized electronic devices. 